\title{
Taxa de geração de resíduos da construção civil em edificações na cidade de João Pessoa
}

\author{
Rate of construction waste generation in buildings in the \\ city of João Pessoa
}

\section{Ricardo Vasconcelos Gomes da Costa Gilson Barbosa Athayde Júnior Mariana Moreira de Oliveira}

\section{Resumo}

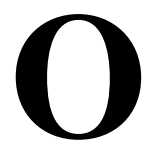

objetivo deste trabalho foi determinar a quantidade de resíduos da construção civil (RCC) em função da área construída da edificação, para efetuar acompanhamento e fiscalização mais eficazes da geração e destinação final em dada obra. Foi escolhida uma amostra representativa das edificações em fase de construção na cidade de João Pessoa. Uma ficha para coleta das características e acompanhamento de volume de RCC descartado ao longo do cronograma de execução da construção foi fornecida aos gestores das edificações; a partir do volume descartado pelas construtoras, determinou-se a massa de RCC gerado em cada obra utilizando uma massa unitária de $1.025 \mathrm{~kg} \mathrm{~m}^{-3}$. Os resultados indicaram uma taxa média de geração de RCC classe A de $86,27 \mathrm{~kg} \mathrm{~m}^{-2}$. Ainda para os RCC classe A, foram definidos os limites inferior e superior com 90\% de confiança para a taxa de geração: 62,31 e $136,02 \mathrm{~kg} \mathrm{~m}^{2}$ respectivamente. A partir desses valores, o controle da geração e disposição de RCC pode ser realizado pelos órgãos competentes, dando o indicativo de quais obras podem estar infringindo a legislação vigente no tocante à destinação de tais resíduos.

Palavras-chave: Resíduos da construção. Quantificação. Gestão de resíduos.

\section{Ricardo Vasconcelos Gomes da \\ Costa \\ Universidade Federal da Paraíba João Pessoa - PB - Brasil}

Gilson Barbosa Athayde Júnior Universidade Federal da Paraíba João Pessoa - PB - Brasil

Mariana Moreira de Oliveira Universidade Federal da Paraíba João Pessoa - PB - Brasil

Recebido em 26/03/12 Aceito em 28/12/13

\section{Abstract}

The aim of this study is to determine the amount of construction waste $(\mathrm{CW})$ based on the built area of buildings, in order to implement more effective monitoring and inspection of the generation and destination of the waste. A representative sample was chosen from buildings under construction in João Pessoa. A form to collect data on the characteristics and $C W$ volume generation of the buildings was distributed. From the volume of $C W$ discharged by the building companies, the weight was calculated based on a unit mass of $1025 \mathrm{~kg} \mathrm{~m}-3$. The results showed a generation rate of class A (CONAMA Resolution 307) CW of $86.27 \mathrm{~kg} \mathrm{~m}-2$. Also, for class A CW, a $90 \%$ confidence interval was calculated, with lower and upper limits of 62.31 and $136.02 \mathrm{~kg} \mathrm{~m}-2$, respectively. Based on these results, the control of the generation and disposal of $\mathrm{CW}$ can be done by local authorities, indicating which sites are not following existing laws on the disposal of waste.

Keywords: Construction waste. Quantification. Waste management. 


\section{Introdução}

A indústria da construção civil vem apresentando altos índices de crescimento no Brasil e trazendo consigo benefícios socioeconômicos, com participação de forma ativa na geração de emprego e renda. Por outro lado, as atividades relacionadas ao setor resultam numa elevada geração de resíduos, os chamados resíduos da construção civil (RCC), que, se dispostos de maneira inadequada, causam impactos ao meio ambiente, como a poluição do solo, assoreamento de córregos, enchentes, proliferação de vetores de doenças e obstrução de vias de tráfego, entre outros. Devido àfrequente falta de informação acerca dos volumes de RCC gerados em determinada obra, faz-se necessário um estudo para o conhecimento da taxa de geração desses resíduos com base na área construída da edificação. Essa taxa poderá servir de parâmetro para o controle e fiscalização dos geradores, visando ao cumprimento das leis e normas vigentes. Este trabalho tem como objetivo estudar a possibilidade de se exprimir a quantidade de RCC em função da área construída da edificação.

\section{RCC: definição, composição e caracterização}

Entre os resíduos gerados no ambiente urbano estão os chamados RCC, que, de acordo com a Resolução no 307 do CONAMA (BRASIL, 2002), são os provenientes de construções, reformas, reparos e demolições de obras de construção, e os resultantes da preparação e da escavação de terrenos, tais como tijolos, blocos cerâmicos, concreto em geral, solos, rochas, metais, resinas, colas, tintas, madeiras e compensados, forros, argamassa, gesso, telhas, pavimento asfáltico, vidros, plásticos, tubulações, fiação elétrica, etc., comumente chamados de entulhos de obras, caliça ou metralha. Ainda de acordo com a Resolução $n^{\circ}$ 307 do CONAMA (BRASIL, 2002), os RCC são classificados em quatro classes distintas:

(a) Classe A: são os resíduos reutilizáveis ou recicláveis como agregados tais como (1) de construção, demolição, reformas e reparos de pavimentação e de outras obras de infraestrutura, inclusive solos provenientes de terraplanagem; (2) de construção, demolição, reformas e reparos de edificações (componentes cerâmicos, argamassa e concreto); e (3) de processo de fabricação e/ou demolição de peças pré-moldadas em concreto (blocos, tubos e meio fio, entre outros), produzidas nos canteiros de obras;

(b) Classe B(nova redação dada pela Resolução CONAMA nº 431 (BRASIL, 2011)): são os resíduos recicláveis para outras destinações, tais como plásticos, papel, papelão, metais, vidros, madeiras e gesso (BRASIL, 2011);

(c) Classe C(nova redação dada pela Resolução CONAMA no 431 (BRASIL, 2011)): são os resíduos para os quais não foram desenvolvidas tecnologias ou aplicações economicamente viáveis que permitam sua reciclagem ou recuperação (BRASIL, 2011); e

(d) Classe $\mathrm{D}$ (nova redação dada pela Resolução CONAMA n 348 (BRASIL, 2004)): são os resíduos perigosos, oriundos do processo da construção, tais como tintas, solventes, óleos e outros, ou aqueles contaminados ou prejudiciais à saúde, oriundos de demolições, reformas e reparos de clínicas radiológicas, instalações industriais e outros, bem como telhas e demais objetos e materiais que contenham amianto ou outros produtos nocivos à saúde (BRASIL, 2004).

As terminologias Resíduos da Construção Civil (RCC) e Resíduos de Construção e Demolição (RCD) têm sido utilizadas no meio acadêmico para denominar os resíduos sólidos gerados nas atividades de construção e demolição. Neste trabalho será utilizado o termo Resíduos da Construção Civil, alinhando-se àquele utilizado na Resolução no 307 do CONAMA (BRASIL, 2002) e já utilizado na literatura (MANFRINATO; ESGUÍCERO; MARTIS, 2008; EVANGELISTA; COSTA; ZANTA, 2010).

A composição e a quantidade dos RCC estão estritamente ligadas às diversas características de sua fonte geradora (construções, reformas, demolições), ao momento de coleta da amostra relativo ao cronograma de execução, bem como à qualidade da mãodeobra, técnicas construtivas empregadas e adoção de programas de qualidade(CARNEIRO, 2005; ZORDAN, 1997). Os RCC gerados no Brasil são compostos predominantemente de materiais inorgânicos não metálicos (em torno de $90 \%$ em massa), consistindo essencialmente de concreto e argamassa, rochas naturais e material cerâmico (ÂNGULO, 2000; ÂNGULO; JOHN, 2006). Com relação ao percentual de RCC classe A, alguns estudos reportam valores na faixa de $88 \%$ a $96 \%$ (BRITO FILHO,1999; COSTA; ALMEIDA, 1999; CARNEIRO et al., 2001; CARNEIRO, 2005;MARQUES NETO; SCHALCH, 2006;MANFRINATO;ESGUÍCERO; MARTIS., 2008), sendo $93 \%$ a média desses valores.

Uma importante característica dos RCC para estudos quantitativos é sua massa unitária, parâmetro este definido como a razão entre sua 
massa e o correspondente volume ocupado, incluindo os vazios. Souza (2005) determinou a massa unitária de RCC no estado bruto gerados na construção de conjuntos habitacionais populares, chegando ao valor de $1.288 \mathrm{~kg} \mathrm{~m}^{-3}$. Carneiro et al.(2000) chegaram ao valor de $1.156 \mathrm{~kg} \mathrm{~m}^{-3}$ na cidade de Salvador, enquanto Ângulo et al. (2011) fazem referência ao valor de $1.000 \mathrm{~kg} \mathrm{~m}^{-3}$. Observa-se que os valores da massa unitária obtidos pelos autores situam-se numa estreita faixa.

\section{Taxas de geração de RCC}

A geração de RCC durante a fase de construção é decorrência das perdas nos processos construtivos (FORMOSO et al., 1998), sendo projetos mal elaborados a principal causa da geração (LLATAS, 2011). Quantitativamente, a geração de RCC é diferente entre várias localidades, devido a diversos fatores, como número de habitantes, leis e regulamentações específicas, processos construtivos, incluindo as peculiaridades de cada construtora (OLIVEIRA et al., 2011), e ainda o momento da construção civil. Assim, essas características interferem diretamente no tipo e na quantidade de resíduo gerado.

A taxa de geração de RCC pode ser definida como a razão entre sua quantidade gerada, geralmente em unidade de volume ou de massa, e outro parâmetro, geralmente o tempo e/ou a população correspondente, ou ainda a área construída.

Vários estudos foram realizados visando obter a taxa de geração de RCC em diversas localidades, tendo sido reportados valores variando de $39 \%$ a $70 \%$ em relação à massa de resíduos sólidos urbanos, $1,2 \times 10^{6}$ a $300 \times 10^{6} \mathrm{t} \mathrm{ano}^{-1}$ ou 0,136 a $3,658 \mathrm{t} \mathrm{hab}^{-1}$ ano $^{-1}$ em alguns países, e ainda $0,20 \mathrm{a}$ $0,76 \mathrm{t} \mathrm{hab}^{-1} \mathrm{ano}^{-1}$ em algumas cidades brasileiras (PINTO, 1999; JOHN, 2000; ATHAYDE JÚNIORet al., 2004; CARNEIRO, 2005; BERNARDES et al., 2008; MANFRINATO;ESGUÍCERO; MARTIS, 2008; VIOLIN, 2009). As taxas supracitadas são de utilização limitada para estimativas futuras de geração de RCC, uma vez que o ritmo da construção civil é extremamente atrelado ao momento econômico e por isso retrata a geração de RCC apenas naquele período correspondente ao estudo.

Por outro lado, taxas reportadas em unidades de massa por unidade de área construída as desvinculam do momento econômico de dada época, além de serem independentes do quantitativo populacional. A seguir, apresentam-se alguns estudos nos quais as taxas de geração de $\mathrm{RCC}$ foram expressas em $\mathrm{kg} \mathrm{m}^{-2}$.
Pinto (1999) considerou uma massa de $1.200 \mathrm{~kg} \mathrm{~m}$ ${ }^{2}$ para edificações finalizadas, executadas predominantemente por processos convencionais, e uma perda média de materiais nos processos construtivos de $25 \%$ em relação à massa de materiais levados ao canteiro de obra, com um percentual de perdas de materiais removido como RCC de 50\%, chegando, assim,à taxa de $150 \mathrm{~kg} \mathrm{~m}$ ${ }^{2}$ de área construída. Monteiro et al. (2001) mencionam valores variando de $100 \mathrm{a} 300 \mathrm{~kg} \mathrm{~m}^{-2}$, porém não indicam os procedimentos metodológicos ou considerações seguidas na obtenção deles.

Carneiro (2005) chegou ao intervalo entre 69,28 e $86,41 \mathrm{~kg} \mathrm{~m} \mathrm{~m}^{-2}$ para a taxa de geração de RCC analisando dados referentes a três canteiros de obra. Para efeito dessa estimativa foram admitidas duas hipóteses para a massa estimada das edificações:

(a) a primeira levando-se em consideração o somatório das cargas de todos os pilares de uma da obra, dividindo-se este valor pela área total construída correspondente e, por fim, subtraindo deste a parcela de carga referente à sobrecarga $\left(150 \mathrm{~kg} \mathrm{~m}^{-2}\right)$, já que esta ainda não existe na etapa de construção, obtendo-se assim um índice de $1.060,29 \mathrm{~kg} \mathrm{~m}^{-2}$; e

(b) a adoção de um valor médio da massa estimada por engenheiros calculistas atuantes na região do estudo, para edificações aporticadas de concreto, de aproximadamente $850 \mathrm{~kg} \mathrm{~m}^{-2}$ (não sendo considerada a parcela da sobrecarga).

Assim, tendo ainda o autor considerado um índice médio de perdas de materiais na forma de RCC de $8,15 \%$ e fazendo o cruzamento de dados entre a massa estimada para a edificação e as perdas, Carneiro (2005) chegou aos valores de 86,41e $69,28 \mathrm{~kg} \mathrm{~m}^{-2}$, baseando-se também, da mesma forma que Pinto (1999), em diversas considerações.

Andrade et al. (2001) fizeram uma estimativa de RCC por unidade de serviço objetivando o cálculo da massa desses resíduos por unidade de área, utilizando-se de indicadores que relacionam a quantidade de serviço executado à área de piso de edificação; finalmente, multiplicando-se os valores de RCC por metro quadrado de piso pela massa de RCC por unidade de material, chegaram ao valor de $49,58 \mathrm{~kg} \mathrm{~m}^{-2}$.

Careli (2008) fez a separação do RCC por tipo de resíduos e assumiu uma estimativa de densidade média por tipo de resíduo considerando a forma como os diferentes tipos de resíduos são acondicionados e coletados, resultando nos valores 
para massa de resíduos de $104,49 \mathrm{~kg} \mathrm{~m}^{-2}$ e 115,82 $\mathrm{kg} \mathrm{m}^{-2}$ para duas obras que foram analisadas.

Solís-Guzman et al. (2009), aplicando o modelo de quantificação utilizando coeficientes estimados de geração de RCC por etapa dos serviços a serem executados, para construção de prédios de quatro andares, com $1.600 \mathrm{~m}^{2}$ de área, chegaram a uma taxa de $307,6 \mathrm{~kg} \mathrm{~m}^{-2}$ considerando o movimento de terra, e de $107,6 \mathrm{~kg} \mathrm{~m}^{-2}$ sem o movimento de terra.Todos os coeficientes foram estimados a partir de dados provenientes da Andalusia Construction Costs Database.

Souza (2005) quantificou os RCC gerados em 50 unidades habitacionais, de 44,52 $\mathrm{m}^{2}$ cada, obtendo o valor de $89,68 \mathrm{~kg} \mathrm{~m}^{-2}$ de RCC gerado através da informação sobre os volumes e uma massa unitária de $1.288 \mathrm{~kg} \mathrm{~m}^{-3}$.

Marques Neto e Schalch (2010) chegaram à taxa de geração quantificando os RCC de cinco obras, sendo uma delas de reforma. Os mesmosautores consideraramuma massa unitária de $600 \mathrm{~kg} \mathrm{~m}^{-3}$, chegando à taxa de $137,02 \mathrm{~kg} \mathrm{~m}^{-2}$. Neste estudo observa-se um valor atípico para a massa unitária, bastante inferior aos valores citados por Carneiro et al. (2000), Souza (2005) e Ângulo et al.( 2011).

Dos estudos acima referidos, apenas Souza (2005) e Marques Neto e Schalch (2010) chegaram à taxa de geração de RCC por unidade de área utilizando um método de medição direta, sendo os demais baseados em estimativas e considerações. Nestes estudos, a área construída a partir da qual os dados foram obtidos foi de 2.226 e $1.795,3 \mathrm{~m}^{2}$ para os estudos de Souza (2005) e Marques Neto e Schalch (2010) respectivamente.

\section{Metodologia}

A pesquisa foi realizada na cidade de João Pessoa, capital do estado da Paraíba, e consistiu no levantamento do volume de RCC descartado em canteiros de obra, com determinação da massa unitária de uma amostra deles e anotação da área construída constante nos projetos correspondentes. Inicialmente, visitaram-se 75 canteiros de obras, dos quais 53 se propuseram a contribuir com informações acerca dos volumes descartados de RCC e das respectivas áreas construídas. Dos 53, apenas 35 possuíam cronograma de execução compatível para ser desenvolvido no período de abril de 2010 a março de 2012. Nessas 35 obras foram incluídas algumas que já se haviam iniciado antes de abril de 2010, mas que ainda não haviam descartado RCC. Reformas não foram consideradas, uma vez que a taxa de geração de RCC esperada deve ser extremamente variável, em função do grau de demolição envolvido. Também não foi computado do volume de RCC proveniente de escavações pelo fato de que tal geração varia bastante, desde quantidades desprezíveis, até volumes representativos no caso da existência de pavimentos no subsolo. Dessa forma, foi considerada apenas a quantificação dos RCC gerados no processo construtivo da edificação.

Para a coleta das características de cada obra e do acompanhamento de volume gerado de RCC, fezse uso, para cada empresa, de uma ficha de acompanhamento e anotação do volume de descarte dos RCC ao longo do cronograma de execução da construção. A lista da amostra de35 obras que fizeram parte desta pesquisa está descrita na Tabela 1, com informações como sua natureza, área a ser construída e período previsto para início e término.

As edificações residenciais referem-se a prédios de apartamentos (obras 1 a 11 e 15) e residências unifamiliares (obras 12 a 14), enquanto as de instituições públicas foram blocos de sala de aula, sala de professores e laboratórios em uma universidade (obras 16 a 33) e clínicas médicas (obras 34 e 35).

\section{Determinação da massa unitária dos RCC}

A massa unitária foi calculada com base em uma amostra dos RCC gerados nas obras 20, 21, 24 e 35 utilizando um recipiente de volume de $0,075575 \mathrm{~m}^{3}$, confeccionado no próprio canteiro de obra, e uma balança com capacidade para $150 \mathrm{~kg}$ e precisão de 0,1 kg, conforme a Equação 1 .

$M_{U}=\frac{M}{V}$

Eq. 1

Onde:

$M_{U}$ : massa unitária do $\mathrm{RCC}\left(\mathrm{kg} \mathrm{m}^{-3}\right)$;

$M$ : massa de $\mathrm{RCC}(\mathrm{kg})$; e

$V$ : volume de $\operatorname{RCC}\left(\mathrm{m}^{3}\right)$.

Foram feitas 20 pesagens, durante as quais foram tomadas as devidas precauções para a obtenção de um valor representativo da massa unitária. Foram coletados RCC das quatro classes descritas na Resolução no 307 do CONAMA (BRASIL, 2002), em etapas construtivas distintas e em períodos secos, para não ocorrer alteração da massa unitária devido à incorporação de umidade. 
Tabela 1- Relação das obras estudadas para o acompanhamento da geração dos RCC

\begin{tabular}{ccccc}
\hline Obra & Natureza & Área construída $\left(\mathbf{m}^{2}\right)$ & Início & Término previsto \\
\hline 1 & Residencial & 985,50 & $\mathrm{jun} / 10$ & $\mathrm{mar} / 11$ \\
2 & Residencial & $12.340,45$ & $\mathrm{ago} / 09$ & $\mathrm{jan} / 12$ \\
3 & Residencial & $43.858,80$ & $\mathrm{dez} / 09$ & $\mathrm{mar} / 12$ \\
4 & Residencial & $16.976,51$ & $\mathrm{set} / 09$ & $\mathrm{jan} / 12$ \\
5 & Residencial & $1.250,00$ & $\mathrm{jan} / 09$ & $\mathrm{ago} / 11$ \\
6 & Residencial & 950,00 & $\mathrm{ago} / 09$ & $\mathrm{jul} / 11$ \\
7 & Residencial & $1.780,00$ & $\mathrm{jul} / 10$ & $\mathrm{ago} / 11$ \\
8 & Residencial & $1.949,00$ & $\mathrm{ago} / 10$ & $\mathrm{out} / 11$ \\
9 & Residencial & $1.194,50$ & $\mathrm{dez} / 09$ & $\mathrm{nov} / 11$ \\
10 & Residencial & 998,00 & $\mathrm{jul} / 09$ & $\mathrm{jul} / 11$ \\
11 & Residencial & $1.450,30$ & $\mathrm{mar} / 09$ & $\mathrm{jan} / 12$ \\
12 & Residencial & 70,00 & $\mathrm{mar} / 11$ & $\mathrm{set} / 11$ \\
13 & Residencial & 95,00 & $\mathrm{jan} / 11$ & $\mathrm{jul} / 11$ \\
14 & Residencial & 150,00 & $\mathrm{fev} / 11$ & $\mathrm{ago} / 11$ \\
15 & Residencial & 595,25 & $\mathrm{jul} / 11$ & $\mathrm{fev} / 12$ \\
16 & Edifício público & $2.299,02$ & $\mathrm{jan} / 09$ & $\mathrm{dez} / 09$ \\
17 & Edifício público & 997,64 & $\mathrm{fev} / 09$ & $\mathrm{mai} / 10$ \\
18 & Edifício público & 312,00 & $\mathrm{jan} / 10$ & $\mathrm{abr} / 12$ \\
19 & Edifício público & 840,00 & $\mathrm{nov} / 10$ & $\mathrm{jul} / 11$ \\
20 & Edifício público & 445,56 & $\mathrm{mar} / 10$ & $\mathrm{fev} / 12$ \\
21 & Edifício público & 232,45 & $\mathrm{abr} / 10$ & $\mathrm{fev} / 12$ \\
22 & Edifício público & 394,30 & $\mathrm{mar} / 10$ & $\mathrm{jan} / 12$ \\
23 & Edifício público & 1154,3 & $\mathrm{abr} / 10$ & $\mathrm{fev} / 12$ \\
24 & Edifício público & 754,93 & $\mathrm{fev} / 11$ & $\mathrm{jan} / 12$ \\
25 & Edifício público & $1.979,00$ & $\mathrm{jan} / 10$ & $\mathrm{jan} / 12$ \\
26 & Edifício público & $1.104,00$ & $\mathrm{fev} / 10$ & $\mathrm{nov} / 12$ \\
27 & Edifício público & $1.399,64$ & $\mathrm{set} / 09$ & $\mathrm{nov} / 12$ \\
28 & Edifício público & $5.170,51$ & $\mathrm{fev} / 10$ & $\mathrm{dez} / 12$ \\
29 & Edifício público & $4.997,00$ & $\mathrm{dez} / 09$ & $\mathrm{nov} / 12$ \\
30 & Edifício público & 850,00 & $\mathrm{set} / 09$ & $\mathrm{jan} / 12$ \\
31 & Edifício público & 474,00 & $\mathrm{mar} / 10$ & $\mathrm{fev} / 12$ \\
32 & Edifício público & 335,00 & $\mathrm{fev} / 10$ & $\mathrm{nov} / 12$ \\
33 & Edifício público & 504,00 & $\mathrm{dez} / 10$ & $\mathrm{nov} / 12$ \\
34 & Edifício público & 455,40 & $\mathrm{mai} / 10$ & $\mathrm{fev} / 11$ \\
35 & Edifício público & $1.031,80$ & $\mathrm{fev} / 10$ & $\mathrm{fev} / 11$ \\
\hline & & & & \\
\hline
\end{tabular}

\section{Levantamento do volume de RCC}

O volume de RCC descartado foi obtido por meio de fichas de acompanhamento distribuídas em cada uma das obras, onde o engenheiro responsável ou outro funcionário de perfil equivalente anotava as quantidades descartadas em contêineres estacionários ou diretamente em caminhões de capacidade volumétrica conhecida. Apesar de o volume gerado de RCC ter sido informado pela própria construtora, nas obras 16, 19, 21, 22, 24 e 35 não se fez necessária a ficha distribuída, e foram escolhidas como obras controle; nestes casos, o pesquisador efetuava visitas diárias nas obras citadas, acompanhando o descarte de RCC e excluindo, portanto, a possibilidade de informação imprecisa por parte da construtora.

\section{Taxa de geração de RCC}

A massa de RCC gerado foi estimadamediantevolumes informados e massa unitária determinada, conforme a Equação 2:

$M=M_{U} V$

Eq. 2

Onde:

$M$ : massa de RCC (kg);

$M_{U}$ : massa unitária do RCC $\left(\mathrm{kg} \mathrm{m}^{-3}\right)$; e

$V$ : volume de $\operatorname{RCC}\left(\mathrm{m}^{3}\right)$.

A taxa de geração dos RCC foi obtida a partir da razão entre sua massa e a área construída de cada obra (Equação 3).

$T x=\frac{M}{A c}$

Eq. 3 
Onde:

$T x$ : taxa de geração de $\mathrm{RCC}\left(\mathrm{kg} \mathrm{m}^{-2}\right)$;

$M$ : massa de RCC (kg); e

$A c$ : área construída $\left(\mathrm{m}^{2}\right)$.

A média ponderada das taxas de geração foi calculada através do somatório da multiplicação das taxas pelas respectivas áreas construídas e posteriormente dividindo-o pelo somatório das áreas construídas (Equação 4).

$\operatorname{Txp}=\frac{\sum_{1}^{n} T x_{i} A c_{i}}{\sum_{1}^{n} A c_{i}}$

Eq. 4

Onde:

Txp: média ponderada das taxas de geração de $\operatorname{RCC}\left(\mathrm{kg} \mathrm{m}^{-2}\right)$;

$T x$ : taxa de geração de $\mathrm{RCC}\left(\mathrm{kg} \mathrm{m}^{-2}\right)$; e

Ac: área construída $\left(\mathrm{m}^{2}\right)$.

Para o cálculo da taxa de RCC classe A foi considerado que os resíduos desta classe representam 93\% no computo geral do RCC, sendo este percentual a média dos valores reportados nos estudos de Brito Filho (1999), Costa e Almeida (1999), Carneiroet al. (2001), Carneiro (2005), Marques Neto e Schalch (2006) eManfrinato, Esguícero e Martis (2008).

\section{Validação da amostra}

Para testar a validade das informações de volume de RCC fornecidas pelas construtoras, foi efetuada análise de variância através do Teste $F$. Esse procedimento estatístico pode ser empregado para testes de hipóteses na comparação das médias de dois ou mais grupos amostrais. Esse teste produz uma estatística ou razão $F$, cujo numerador representa a variação entreos grupos $\left(S_{b}^{2}\right)$, e cujo denominador contém uma estimativa da variação dentrodos grupos $\left(S_{w}^{2}\right)$. A formulação matemática do teste (Eq. 5, Eq. 6 e Eq. 7) encontra-se descrita em Lapponi (1995). Calcula-se o valor de $F$ pela relação mostrada na Equação 5:

$$
F=\frac{S_{b}^{2}}{S_{w}^{2}}
$$

Onde:

$F$ : estatística $F$;

$S_{b}^{2}$ : variância entre os grupos; e

$S_{w}^{2}$ : variância dentro dos grupos.

A variância entre os grupos é calculada através da Equação 6:
$S_{b}^{2}=\frac{\sum_{1}^{k} n_{j}\left(\bar{X}_{j}-\overline{\bar{X}}\right)^{2}}{k-1}$

Onde:

$S_{b}^{2}$ : variância entre os grupos;

$n$ : número de elementos do grupo;

$\bar{X}$ : média do grupo; e

$\overline{\bar{X}}$ : grande média.

A grande média é a média de todas as observações.

Avariância dentroé calculada através da Equação 7:

$S_{w}^{2}=\frac{\sum_{1}^{k}\left(n_{j}-1\right) S_{j}^{2}}{n_{T}-k}$ Eq. 7

Onde:

$S_{w}^{2}$ : variância dentro dos grupos;

$n$ : número de elementos do grupo;

$S$ : variância do grupo;

$n_{T}$ : número total de observações; e

$k$ : número de grupos.

Calculado o valor de $F$, compara-sea estatística com o valor $F_{C}$ (F crítico) da distribuição $F$, que será determinado a partir da relação do grau de liberdade da variável do numerador $\left(v_{1}=k-1\right) \mathrm{e}$ grau de liberdade da variável do denominador $\left(v_{2}=\right.$ $\left.n_{\mathrm{T}}-k\right)$ para dado nível de significância. Se o valor da estatística $F$ for menor que o valor crítico da distribuição $F$, diz-se que as médias amostrais não são significativamente diferentes entre si, e se o valor da estatística $F$ for maior que o valor crítico da distribuição $F$, diz-se que as médias amostrais são significativamente diferentes entre si (LAPPONI, 1995). Os grupos analisados foram o grupo 1, formado pelas obras controle, e o grupo 2, formado pelas demais obras. O nível de significância estatística $(\alpha)$ adotado neste estudo foi de $5 \%$.

\section{Resultados e discussão}

\section{Massa unitária do RCC}

A média da massa unitária e seus limites de $95 \%$ de confiança (Figura 1) foram comparados com valores reportados na literatura (CARNEIROet al., 2000; SOUZA, 2005; ÂNGULO et al., 2011). A média aritmética da massa unitária das 20 amostras foi de $1.025 \mathrm{~kg} \mathrm{~m}^{-3}$, valor que se aproxima dos obtidos por Carneiro et al. (2000), Souza (2005) e Ângulo et al. (2011). O valor médio encontrado 
$\left(1025 \mathrm{~kg} \mathrm{~m}^{-3}\right)$ foi utilizado no cálculo da taxa de geração de RCC.

\section{Taxa de geração de RCC}

Das 35 obras consideradas, foram utilizadas para o cálculo da taxa de geração de RCC apenas aquelas finalizadas até 23 de março de 2012, que corresponderam a 22 obras. Juntas, as 22 obras somaram uma área construída de $82.705,41 \mathrm{~m}^{2}$, valor superior aos reportados por Souza (2005) e Marques Neto e Schalch (2010). A Tabela 2 apresenta,para as 22 obras finalizadas, os valoresda área construída, volume de RCC gerado,massa de RCC gerada(calculada utilizando a Equação 2 e o valor da massa unitária de $1025 \mathrm{~kg} \mathrm{~m}^{-3}$ ) e taxa de geração de RCC, calculada através da Equacção 3.

A análise de variância através do Teste $\mathrm{F}$, comparando a taxa média do grupo 1 (obras controle) e a taxa média do grupo 2 (demais obras), foi efetuada e os resultados estão sumarizados nas Tabelas3 e 4. Foi calculado o valor de $F$ igual a 0,00041 , que, comparado ao valor de $F_{C}$ igual a 4,35 , permite concluir que não há diferença significativa entre as médias das taxas de geração de RCC das obras controle e das demais obras, e portanto não há razão para se acreditar em informação imprecisa por parte das construtoras quanto aos dados de geração de RCC.

Figura 1-Valores comparativos da massa unitária de RCC

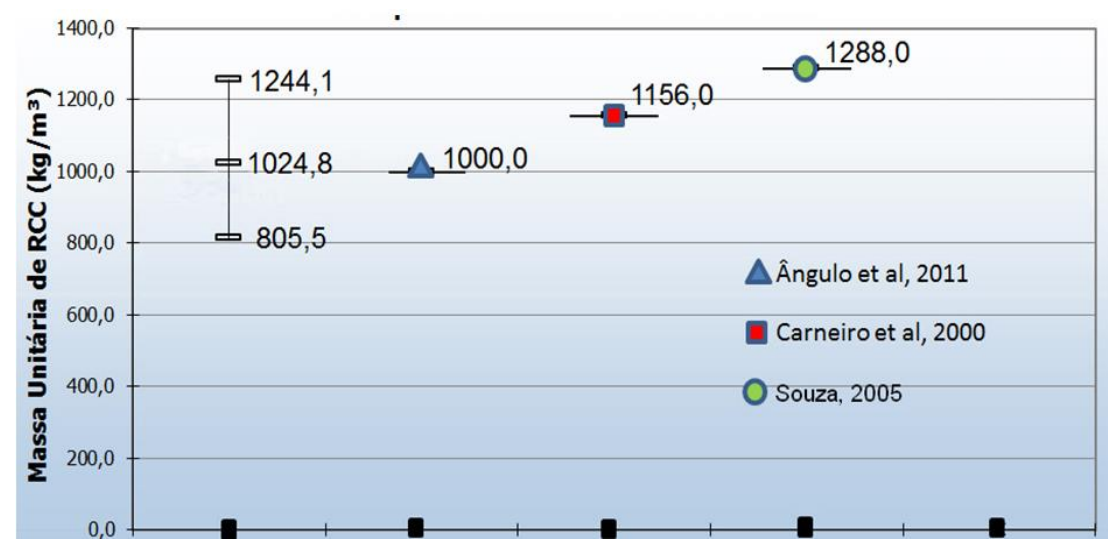

Tabela 2 - Relação das obras estudadas, com geração de RCC

\begin{tabular}{|c|c|c|c|c|c|c|}
\hline Obra & Natureza & $\begin{array}{c}\text { Área } \\
\text { construída } \\
\left(\mathbf{m}^{2}\right)\end{array}$ & $\begin{array}{c}\text { Volume } \\
\text { gerado de } \\
\operatorname{RCC}\left(\mathbf{m}^{3}\right)\end{array}$ & $\begin{array}{c}\text { Massa gerada } \\
\text { de } \mathrm{RCC}(\mathrm{kg})\end{array}$ & $\begin{array}{l}\text { Taxa de geração } \\
\text { de } \operatorname{RCC}\left(\mathrm{kg} \mathrm{m}^{-2}\right)\end{array}$ & $\begin{array}{c}\text { Taxa de geração } \\
\text { de RCC classe A } \\
\left(\mathrm{kg} \mathrm{m}^{-2}\right)\end{array}$ \\
\hline 1 & Residencial & 985,50 & 97,0 & $99.425,00$ & 100,89 & 93,83 \\
\hline 3 & Residencial & $43.858,80$ & $3.854,5$ & $3.950 .862,50$ & 90,08 & 83,78 \\
\hline 4 & Residencial & $16.976,51$ & $1.515,5$ & $1.553 .387,50$ & 91,50 & 85,10 \\
\hline 5 & Residencial & $1.250,00$ & 120,0 & $123.000,00$ & 98,40 & 91,51 \\
\hline 6 & Residencial & 950,00 & 100,0 & $102.500,00$ & 107,89 & 100,34 \\
\hline 7 & Residencial & $1.780,00$ & 140,0 & $143.500,00$ & 80,62 & 74,98 \\
\hline 8 & Residencial & $1.949,00$ & 180,0 & $184.500,00$ & 94,66 & 88,03 \\
\hline 9 & Residencial & $1.194,50$ & 144,0 & $147.600,00$ & 123,57 & 114,92 \\
\hline 10 & Residencial & 998,00 & 105,0 & $107.625,00$ & 107,84 & 100,29 \\
\hline 12 & Residencial & 70,00 & 10,0 & $10.250,00$ & 146,43 & 136,18 \\
\hline 13 & Residencial & 95,00 & 10,5 & $10.762,50$ & 113,29 & 105,36 \\
\hline 14 & Residencial & 150,00 & 11,0 & $11.275,00$ & 75,17 & 69,91 \\
\hline 16 & Edifício público & $2.299,02$ & 203,10 & $208.177,50$ & 90,55 & 84,21 \\
\hline 17 & Edifício público & 997,64 & 165,5 & $169.586,25$ & 169,99 & 158,09 \\
\hline 19 & Edifício público & 840,00 & 72,00 & $73.800,00$ & 87,86 & 81,71 \\
\hline 20 & Edifício público & 445,56 & 48,5 & $49.712,50$ & 111,57 & 103,76 \\
\hline 21 & Edifício público & 232,45 & 36,0 & $36.900,00$ & 158,74 & 147,63 \\
\hline 22 & Edifício público & 394,30 & 38,5 & $39.462,50$ & 100,08 & 93,08 \\
\hline 24 & Edifício público & 754,93 & 64,50 & $66.112,50$ & 87,57 & 81,44 \\
\hline 29 & Edifício público & $4.997,00$ & 508,3 & $521.007,50$ & 104,26 & 96,96 \\
\hline 34 & Edifício público & 455,40 & 39,5 & $40.487,50$ & 88,91 & 82,69 \\
\hline 35 & Edifício público & $1.031,80$ & 100,40 & $102.910,00$ & 99,74 & 92,76 \\
\hline Total & - & $82.705,41$ & $7.575,75$ & $7.765 .143,75$ & - & - \\
\hline
\end{tabular}


Tabela 3-Estatística descritiva da taxa de geração de RCC para as obras controle (grupo 1) e demais obras (grupo 2)

\begin{tabular}{lccccc}
\hline & $\boldsymbol{n}$ & Soma & $\bar{X}$ & $\boldsymbol{S}$ & $\boldsymbol{S}^{2}$ \\
\hline Grupo 1 & 6 & 640,84 & 106,81 & 26,17 & 684,83 \\
Grupo 2 & 16 & 1705,07 & 106,57 & 24,07 & 579,33 \\
\hline
\end{tabular}

Tabela 4 -Análise de variância dataxa de geração de RCC para os grupos 1 e 2

\begin{tabular}{ccccccccc}
\hline $\boldsymbol{k}$ & $\boldsymbol{n}_{\boldsymbol{T}}$ & $\overline{\overline{\boldsymbol{X}}}$ & $\boldsymbol{S}_{\boldsymbol{w}}^{\mathbf{2}}$ & $\boldsymbol{S}_{\boldsymbol{b}}^{\mathbf{2}}$ & $\boldsymbol{F}$ & $\boldsymbol{v}_{\mathbf{1}}$ & $\boldsymbol{v}_{\mathbf{2}}$ & $\boldsymbol{F}_{\boldsymbol{C}}$ \\
\hline 2 & 22 & 106,63 & 605,70 & 0,25 & 0,00041 & 1 & 20 & 4,35 \\
\hline
\end{tabular}

Das obras concluídas (22 obras), 12 são residenciais e 10 são de instituições públicas. Foi efetuada análise de variância também para se verificarem possíveis diferenças entre as médias das taxas de geração das obras de natureza residencial (grupo 3) e das obras de edifícios públicos (grupo 4). Comparando-se o valor calculado de $F(0,762)$ com o valor de $F_{C}(4,35)$, conclui-se que não há diferenças significativas nas médias das taxas das obras de edificações residenciais e das obras de edifícios públicos (ver Tabelas 5 e 6).

Dessa forma, considerou-se a taxa de geração de RCC de todas as obras concluídas sem distinção quanto à tipologia de edificação residencial ou edifício público. A média aritmética das taxas de geração de RCC das obras concluídas foi de $106,63 \mathrm{kgm}^{-2}$. Considerando a média ponderada das taxas de geração, com a área construída de cada obra sendo o peso na ponderação, o valor médio passa a ser $93,89 \mathrm{kgm}^{-2}$. Observa-se que esse valor é próximo a 89,68 $\mathrm{kgm}^{-2}$ (SOUZA, 2005).

Considerando resíduos apenas de classe A,tem-se que a taxa média aritmética foi de $99,17 \mathrm{kgm}^{-2}$, e a ponderada (sendo o peso na ponderação a área construída) de $86,27 \mathrm{kgm}^{-2}$. A partir da taxa de RCC classe A encontrada (média aritmética), foram calculadosos limites superior e inferior com 90\% deconfiança, mostrados na Tabela 7.

Dessa forma, considerou-se a taxa de geração de RCC de todas as obras concluídas sem distinção quanto à tipologia de edificação residencial ou edifício público. A média aritmética das taxas de geração de RCC das obras concluídas foi de $106,63 \mathrm{kgm}^{-2}$. Considerando a média ponderada das taxas de geração, com a área construída de cada obra sendo o peso na ponderação, o valor médio passa a ser $93,89 \mathrm{kgm}^{-2}$. Observa-se que esse valor é próximo a 89,68 $\mathrm{kgm}^{-2}$ (SOUZA, 2005).

Considerando resíduos apenas de classe A,tem-se que a taxa média aritmética foi de $99,17 \mathrm{kgm}^{-2}$, e a ponderada (sendo o peso na ponderação a área construída) de $86,27 \mathrm{kgm}^{-2}$. A partir da taxa de RCC classe A encontrada (média aritmética), foram calculadosos limites superior e inferior com 90\% deconfiança, mostrados na Tabela 7.

Como recomendação, os limites superior e inferior citados na Tabela 7 podem ser utilizados para fins de fiscalização e de gerenciamento junto a dada obra. Caso o gestor de uma obra declare uma quantidade gerada de RCC que resulte numa taxa inferior a $62,31 \mathrm{kgm}^{-2}$, o mesmodeveria pedir esclarecimentosao órgão fiscalizador, demonstrando a baixa geração de RCC; esse procedimento poderia contribuir para a diminuição de deposições irregulares de RCC. No caso das obras que apresentem taxas de geração dos RCC acima de 136,02 $\mathrm{kg} \mathrm{m}^{-2}$, o gerenciamento dos processos construtivos empregados nessa obra deve ser revisto, de modo a minimizar tal geração e a evitar o desperdício na construção civil. 
Tabela 5-Estatística descritiva da taxa de geração de RCC para as obras de edificações residenciais (grupo 3) e obrasde edifícios públicos (grupo 4)

\begin{tabular}{cccccc}
\hline & $\boldsymbol{n}$ & Soma & $\bar{X}$ & $\boldsymbol{S}$ & $\boldsymbol{S}^{\mathbf{2}}$ \\
\hline Grupo 3 & 12 & 1230,34 & 102,53 & 19,38 & 375,41 \\
Grupo 4 & 10 & 1115,57 & 111,56 & 28,94 & 837,79 \\
\hline
\end{tabular}

Tabela 6 -Análise de variância dataxa de geração de RCC para os grupos 3 e 4

\begin{tabular}{ccccccccc}
\hline $\boldsymbol{k}$ & $\boldsymbol{n}_{\boldsymbol{T}}$ & $\overline{\overline{\boldsymbol{X}}}$ & $\boldsymbol{S}_{\boldsymbol{w}}^{\mathbf{2}}$ & $\boldsymbol{S}_{\boldsymbol{b}}^{\mathbf{2}}$ & $\boldsymbol{F}$ & $\boldsymbol{v}_{\mathbf{1}}$ & $\boldsymbol{v}_{\mathbf{2}}$ & $\boldsymbol{F}_{\boldsymbol{C}}$ \\
\hline 2 & 22 & 106,63 & 583,48 & 444,64 & 0,762 & 1 & 20 & 4,35 \\
\hline
\end{tabular}

Tabela 7-Média aritmética, desvio padrão e limites de confiança para a taxa de geração de RCC classe A

\begin{tabular}{lc}
\hline Taxa média dos RCC Classe $\mathrm{A}\left(\mathrm{kg} \mathrm{m}^{-2}\right)$ & 99,17 \\
\hline Desvio padrão $\left(\mathrm{kg} \mathrm{m}^{-2}\right)$ & 22,34 \\
Limite superior com $90 \%$ de confiança $\left(\mathrm{kg} \mathrm{m}^{-2}\right)$ & 136,02 \\
Limite inferior com $90 \%$ de confiança $\left(\mathrm{kg} \mathrm{m}^{-2}\right)$ & 62,31 \\
\hline
\end{tabular}

\section{Conclusões}

A taxa de RCC bruto (classes A, B, C e D) encontrada em edificações na cidade de João Pessoa foi de $93,89 \mathrm{~kg} \mathrm{~m}^{-2}$ de área construída.

A taxa média encontrada para os RCC de classe A foi de $86,27 \mathrm{~kg} \mathrm{~m}^{-2}$, sendo os limites com $90 \%$ de confiança inferior e superior para a média $62,31 \mathrm{~kg}$ $\mathrm{m}^{-2}$ e $136,02 \mathrm{~kg} \mathrm{~m}^{-2}$ respectivamente.

\section{Referências}

ANDRADE, A. C. et al. Estimativa da Quantidade de Entulho Produzido em Obras de Construção de Edifícios. In: SEMINÁRIO DESENVOLVIMENTO SUSTENTÁVEL E A RECICLAGEM NA CONSTRUÇÃO CIVIL; MATERIAIS RECICLADOS E SUAS APLICAÇÕES, 4., São Paulo, 2001. Anais... São Paulo, 2001.

ÂNGULO, S. C. Variabilidade de Agregados Graúdos de Resíduos da Construção e Demolição Reciclados. São Paulo, 2000.155 f.Dissertação (Mestrado em Engenharia Civil) Escola Politécnica, Universidade de São Paulo, São Paulo, 2000.

ÂNGUlO, S. C.; JOHN, V. M. Requisitos Para Execução de Aterros de Resíduos de Construção e Demolição. Boletim Técnico da Escola Politécnica da USP, Departamento de Engenharia de Construção Civil: BT/PCC/436. Escola Politécnica da Universidade de São Paulo. São Paulo, 2006.
ÂNGULO, S. C. et al. Resíduos de Construção e Demolição: avaliação de métodos de quantificação. Engenharia Sanitária e Ambiental, Rio de Janeiro, v. 16, n. 3, p. 299-306, 2011.

ATHAYDE JÚNIOR, G. B. et al.Reciclagem de Entulhos em Governador Valadares: uma alternativa viável. In: CONGRESSO BRASILEIRO DE CIÊNCIA E TECNOLOGIA EM RESÍDUOS E DESENVOLVIMENTO SUSTENTAVÉL, Florianópolis, 2004. Anais... Florianópolis, 2004.

BERNARDES, A. et al. Quantificação e Classificação dos Resíduos de Construção e Demolição Coletados no Município de Passo Fundo, RS. Ambiente Construído, Porto Alegre, v. 8, n. 3, p. 65-76, jul./set. 2008.

BRASIL. Ministério do Meio Ambiente. Resolução CONAMA n. 307, de 5 de julho de 2002, estabelece diretrizes, critérios e procedimentos para a gestão de resíduos da construção civil. 2002. Diário Oficial da União, n. 136, de 17 de julho de 2002, Seção 1, p. 95-96.

BRASIL. Resolução CONAMA no 348 , de 16 de agosto de 2004. Diário Oficial República Federativa do Brasil. Conselho Nacional do Meio Ambiente. Brasília, DF, 2004.

BRASIL. Ministério do Meio Ambiente. Resolução CONAMA n. 431, de 24 de maio de 2011, que altera o artigo $3^{\circ}$ da Resolução ${ }^{\circ} 307$. 2011. Diário Oficial da União, n. 96, de 25 de maio de 2011. 
BRITO FILHO, J. A. Cidade Versus Entulhos. In: SEMINÁRIO SOBRE DESENVOLVIMENTO SUSTENTÁVEL E A RECICLAGEM NA CONSTRUÇÃO CIVIL, 2., São Paulo, 1999. Anais... São Paulo: IBRACON, 1999. p. 56-67.

CARELI, E. D. A Resolução CONAMA no 307/2002 e as novas condições para gestão dos resíduos de construção e demolição. São Paulo, 2008. 154 f. Dissertação (Mestrado em Engenharia Civil) - Centro Estadual de Educação Tecnológica Paula Souza, São Paulo, 2008.

CARNEIRO, A. P. et al. Reciclagem de Entulho da Região Metropolitana de Salvador Para a Produção de Materiais de Construção de Baixo Custo. In: SIMPÓSIO LUSO-BRASILEIRO DE ENGENHARIA SANITÁRIA E AMBIENTAL, 9., Porto Seguro, 2000. Anais... Porto Seguro: ABES, 2000.

CARNEIRO, A. P. et al. Características do Entulho e do Agregado Reciclado. In: CASSA, J. C. S.; CARNEIRO, A. P.; BRUN, I. A. S. (Orgs.). Reciclagem de Entulhos Para a Produção de Materiais de Construção: Projeto Entulho Bom. Salvador: EDUFBA - CEF, 2001.

CARNEIRO, F. P. Diagnóstico e Ações da Atual Situação dos Resíduos de Construção e Demolição na Cidade do Recife. João Pessoa, 2005. 131 f. Dissertação (Mestrado em Engenharia Urbana) - Escola de Engenharia, Universidade Federal da Paraíba, João Pessoa, 2005.

COSTA, L. S. N.; ALMEIDA, S. L. M. Caracterização Tecnológica dos Resíduos de Construção e Demolição (RCD) da Cidade de Macaé - RJ. In: JORNADA DO PROGRAMA DE CAPACITAÇÃO INTERNA - CETEM,Rio de Janeiro, 1999. Anais... Rio de Janeiro: CETEM/MCT, 1999.

EVANGELISTA, P. P. A.; COSTA, D. B.; ZANTA, V. M.Alternativa Sustentável Para Destinação de Resíduos de Construção Classe A: sistemática para reciclagem em canteiros de obras. Ambiente Construído, Porto Alegre, v. 10, n. 3, p. 23-40, jul./set. 2010.

FORMOSO, C. T. et al. Perdas de Materiais na Construção Civil: um estudo em canteiros de obras no Estado do Rio Grande do Sul. In: CONGRESSO LATINO AMERICANO DE TECNOLOGIA E GESTÃO NA PRODUÇÃO DE EDIFÍCIOS: SOLUÇÕES PARA O TERCEIRO MILÊNIO, São Paulo, 1998. Anais... São Paulo: POLI-USP, 1998. p. 299-307.
JOHN, V. M. Reciclagem de Resíduos na Construção Civil: contribuição à metodologia de pesquisa e desenvolvimento. São Paulo, 2000. 102 f. Tese (Livre docência) - Escola Politécnica, Universidade de São Paulo, São Paulo, 2000.

LAPPONI, J. C. Estatística Usando EXCEL. São Paulo: Lapponi Treinamento e Editora Ltda., $1995.292 \mathrm{p}$.

LLATAS, C. A. A model For Quantifying Construction Waste in Projects According to the European Waste List.Waste Management, v. 31, n. 6, p. 1261-1276, 2011.

MANFRINATO, J. W. S.; ESGUÍCERO, F. J.; MARTIS, B. L. Implementação de Usina Para Reciclagem de Resíduos da Construção Civil (RCC) Como Ação Para o Desenvolvimento Sustentável: estudo de caso. In: ENCONTRO NACIONAL DE ENGENHARIA DE PRODUÇÃO,28., Rio de Janeiro, 2008. Anais... Rio de Janeiro, 2008.

MARQUES NETO, J. C.; SCHALCH, V. Diagnóstico Ambiental Para Gestão Sustentável dos Resíduos de Construção e Demolição. In:SIMPÓSIO LUSO-BRASILEIRO DE ENGENHARIA SANITÁRIA E AMBIENTAL, 12.,Figueira da Foz, Portugal, 2006. Anais... Filgueira da Foz, Portugal: APRH/ABES, 2006.

MARQUES NETO, J. C.; SCHALCH, V. Gestão dos Resíduos de Construção e Demolição: Estudo da Situação no Município de São Carlos, SP.

Engenharia Civil, Minho, Portugal, v. 36, p. 4150, 2010.

MONTEIRO, J. H. P. et al.Manual de Gerenciamento Integrado de Resíduos Sólidos. Rio de Janeiro: IBAM, 2001.

OLIVEIRA, M. M. et al. Determinação da Taxa de Geração de RCC: estudo de caso das obras do campus I da UFPB. In: CONGRESSO BRASILEIRO DE ENGENHARIA SANITÁRIA E AMBIENTAL, 26., Porto Alegre, 2011. Anais... Porto Alegre, 2011.

PINTO, T. P.Metodologia Para a Gestão Diferenciada de Resíduos Sólidos da Construção Urbana. São Paulo, 1999.189 f. Tese (Doutorado em Engenharia Civil) - Escola Politécnica, Universidade de São Paulo, São Paulo, 1999.

SOLÍS-GUZMAN, J. et al.A Spanish Model For Quantification and Management of Construction Waste. Waste Management, v. 29, n. 9, p. 2542 2548, 2009 
SOUZA, V. B. Avaliação da Geração de

Entulho em Conjunto Habitacional

Popular:estudo de caso. Uberlândia, 2005. $251 \mathrm{f}$.

Dissertação (Mestrado em Engenharia Civil) -

Faculdade de Engenharia Civil, Universidade

Federal de Uberlândia, Uberlândia, 2005.

VIOLIN, R. Y. T. Diagnóstico da Geração de

Resíduos de Construção e Demolição em Etapas

Construtivas no Município de Maringá/PR.

Maringá, 2009. 116 f. Dissertação (Mestrado) -

Programa de Pós-Graduação em Engenharia

Urbana, Universidade Estadual de Maringá,

Maringá, 2009.
ZORDAN, S. E. A Utilização do Entulho Como Agregado na Confecção do Concreto. Campinas, 1997. 140 f. Dissertação (Mestrado em Engenharia Civil) - Departamento de Saneamento e Meio Ambiente da Faculdade de Engenharia Civil, Universidade Estadual de Campinas, Campinas, 1997.

\section{Revista Ambiente Construído}

Associação Nacional de Tecnologia do Ambiente Construído

Av. Osvaldo Aranha, $99-3^{\circ}$ andar, Centro

Porto Alegre - RS - Brasil

CEP $90035-190$

Telefone: +55 (51) 3308-4084

Fax: +55 (51) 3308-4054

www.seer.ufrgs.br/ambienteconstruido

E-mail: ambienteconstruido@ufrgs.br 